\title{
Peripheral Antinociception by Carbamazepine in an Inflammatory Mechanical Hyperalgesia Model in the Rat: a New Target for Carbamazepine?
}

\author{
Sonja Vučković ${ }^{1, *}$, Maja Tomić ${ }^{2}$, Radica Stepanović-Petrović ${ }^{2}$ Nenad Ugrešić ${ }^{2}$, Milica Prostran ${ }^{1}$, \\ and Bogdan Bošković ${ }^{3}$ \\ ${ }^{I}$ Department of Pharmacology, Clinical Pharmacology and Toxicology, Faculty of Medicine, University of Belgrade, \\ Dr Subotića 1, P.O. Box 840, 11129 Belgrade, Serbia and Montenegro \\ ${ }^{2}$ Department of Pharmacology, Faculty of Pharmacy, University of Belgrade, P.O. Box 146, Vojvode Stepe 450, \\ 11221 Belgrade, Serbia and Montenegro \\ ${ }^{3}$ Medical Military Academy, Crnotravska 17, 11000 Belgrade, Serbia and Montenegro
}

Received November 7, 2005; Accepted January 31, 2006

\begin{abstract}
This study investigated whether carbamazepine could produce local peripheral antinociception in a rat model of inflammatory mechanical hyperalgesia, and whether adenosine receptors are involved. Carbamazepine $(100-1000 \mathrm{nmol} / \mathrm{paw})$ coadministrated with a proinflammatory compound, concanavalin $\mathrm{A}$, into the hind paw caused a significant dose- and time-dependent anti-hyperalgesia. Coadministration of caffeine $(250-1000 \mathrm{nmol} / \mathrm{paw})$, a nonselective adenosine-receptor antagonist, as well as DPCPX $(10-30 \mathrm{nmol} / \mathrm{paw})$, a selective adenosine $\mathrm{A}_{1}$-receptor antagonist, with carbamazepine, significantly depressed its antihyperalgesic effect. Drugs injected into the contralateral hind paw did not produce significant effects. These results suggest that carbamazepine produces local peripheral anti-hyperalgesia via peripheral adenosine $A_{1}$ receptors.
\end{abstract}

Keywords: local carbamazepine, inflammatory mechanical hyperalgesia, adenosine $\mathrm{A}_{1}$ receptor

Carbamazepine is a well known anticonvulsant drug that has been widely used as an analgesic in the treatment of neuropathic pain. In recent years, increasing evidence appeared that carbamazepine is effective in different animal models of pain, but the sites and mechanisms of its action are not completely understood $(1-5)$.

In our previous study, we have demonstrated that systemic carbamazepine reversed the hyperalgesia induced by intraplantar administration of a pro-inflammatory compound, concanavalin A (Con A), into the rat hind paw and that this effect is mediated via adenosine $A_{1}$ receptors (4). As it is well known that activation of both central and peripheral adenosine $A_{1}$ receptors inhibits pain in rodents (6), this finding raised further questions related to the potential involvement of

*Corresponding author. svuckovic@med.bg.ac.yu

Published online in J-STAGE: March 31, 2006

DOI: $10.1254 /$ jphs.SCE05003X the peripheral adenosine receptors in the carbamazepineinduced antinociception. The interaction of carbamazepine with central adenosine receptors has already been demonstrated in receptor binding studies $(1,7)$, as well as in a behavioral study of nociception (8). On the other hand, the interaction of carbamazepine with adenosine receptors in peripheral pain pathways has not been evaluated before.

The present study had two objectives. First, to determine the effect of locally administered carbamazepine on inflammatory mechanical hyperalgesia in the hind paw of the rat. Second, to determine a potential involvement of adenosine receptors in the local peripheral action of carbamazepine by assessing the effect of caffeine (a nonselective adenosine $\mathrm{A}_{1}$ and $\mathrm{A}_{2}$ receptor antagonist) and 1,3-dipropyl-8-cyclopentylxanthine (DPCPX) (a selective adenosine $\mathrm{A}_{1}$-receptor antagonist) on carbamazepine-induced antinociception.

Groups of $6-8$ male Wistar rats $(180-220 \mathrm{~g})$ were used. The investigation conforms with the Guide for the 


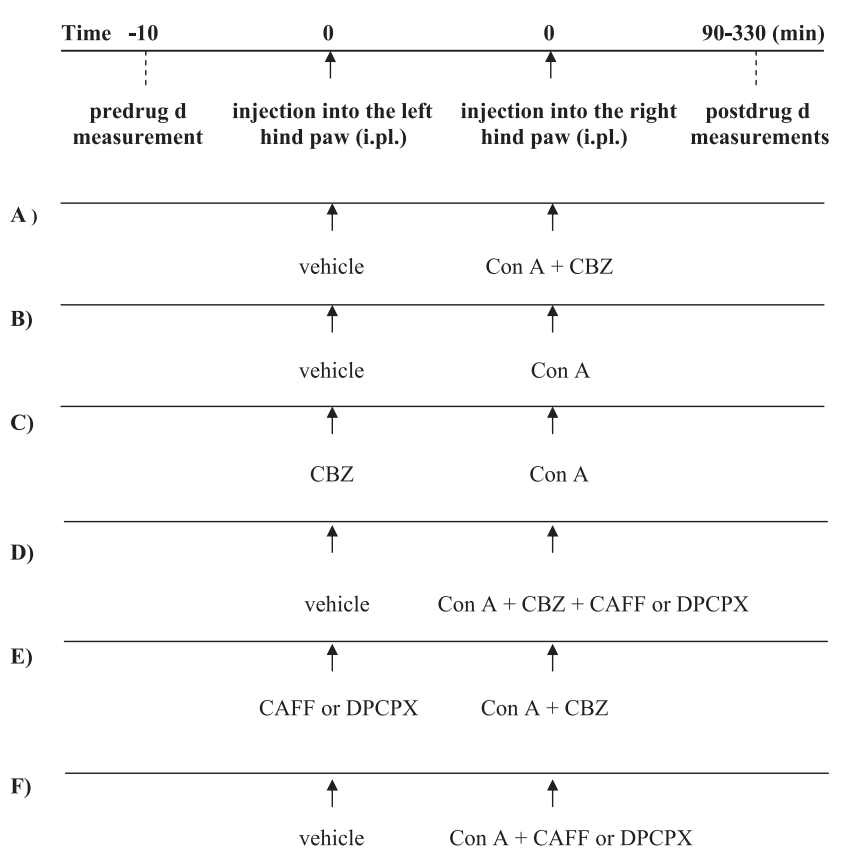

Fig. 1. Experimental protocol used in the evaluation of antihyperalgesic effect of intraplantar (i.pl.) carbamazepine (CBZ) coadministrated with concanavalin A (Con A) (A), hyperalgesia induced by i.pl. injection of Con A (B), effect of CBZ injected contralaterally (C), the influence of caffeine (CAFF) or 1,3-dipropyl8-cyclopentylxantine (DPCPX) coadministrated with Con A + CBZ on the anti-hyperalgesic effect of CBZ (D), the influence of CAFF or DPCPX injected contralaterally on the anti-hyperalgesic effect of CBZ (E), and the influence of CAFF or DPCPX on Con A induced hyperalgesia $(\mathrm{F}) . \mathrm{d}=$ difference in pressures (in grams) applied to left and right hind paws. Predrug d was obtained $10 \mathrm{~min}$ before intraplantar injection. The right hind paw was injected immediately after the left one.

Care and Use of Laboratory Animals published by the U.S. National Institute of Health (NIH Publication No. $85-23$, revised 1985).

Induction of inflammation and the paw pressure test was performed as previously described (4). Briefly, the rat was placed with its hind paws on two transducer platforms of the apparatus (Hugo Sachs Elektronik, March-Hugstetten, Germany) and pushed slowly and smoothly downwards, until one of the paws exceeds the trigger level set at $100 \mathrm{~g}$. The difference (d) in pressures (in grams) applied to non-inflamed (vehicleinjected) and inflamed (Con A-injected) rat hind paw is determined after each measurement. Firstly, in order to examine the peripheral effect of carbamazepine, the drug and the Con A were coadministrated intraplantarly (i.pl.), into the right hind paw (Fig. 1A). Control animals received the same volume of Con A (i.pl.) dissolved in the same vehicle (Fig. 1B). To exclude the possible systemic effect of intraplantarly injected drug, the highest dose of carbamazepine used was given contralat- erally (Fig. 1C). Next, the influences of caffeine and DPCPX on the peripheral anti-hyperalgesic action of carbamazepine were tested. Antagonists were coadministrated with carbamazepine and Con A, intraplantarly, into the right hind paw (Fig. 1D). The comparative group of animals received the same volume of Con A with carbamazepine (Fig. 1A). To exclude the possible systemic effect of intraplantarly injected caffeine or DPCPX, the highest tested dose of each antagonist was given contralaterally (Fig. 1E). Finally, the effects of coadministration of the highest doses of caffeine and DPCPX used with Con A (Fig. 1F) have been evaluated and compared with the effect of Con A alone (Fig. 1B). Carbamazepine (Sigma-Aldrich Chemie $\mathrm{GmbH}$, Munich, Germany), caffeine (Galenika, Belgrade, Serbia and Montenegro), and DPCPX (Sigma-Aldrich Chemie $\mathrm{GmbH}$ ) were dissolved or suspended in a vehicle containing 50\% polyethylene glycol 400 (PEG 400) and $50 \%$ saline and sonicated for $15 \mathrm{~min}$ for proper distribution. Con A was used in a fixed dose of 0.8 $\mathrm{mg} / \mathrm{paw}$. All substances were injected intraplantarly in a final volume of $0.1 \mathrm{ml} / \mathrm{paw}$. Results are expressed as means \pm S.E.M. Calculations for percent antihyperalgesic activity (\%AA), percent inhibition of antihyperalgesic activity $(\% \mathrm{I})$, and $\mathrm{ED}_{50}$ value were done according to references 4,9 , and 10 . Statistical comparisons were made by Student's $t$-test or analysis of variance (One-Way ANOVA), followed by Tukey's HSD test (10). A $P<0.05$ was considered significant.

The coadministration of carbamazepine $(100-1000$ nmol/paw, i.pl.) with Con A produced a significant dose- and time-dependent reduction in differences in forces exerted by inflamed and non-inflamed rat hind paw in a paw pressure test (Fig. 2A). The least $\mathrm{ED}_{50}$ value $(95 \%$ confidence limits) of $920(512-1654)$ $\mathrm{nmol} / \mathrm{paw} \quad(0.217 \mathrm{mg} / \mathrm{paw})$ was obtained $150 \mathrm{~min}$ after i.pl. injection. Coadministration of caffeine (250 and $1000 \mathrm{nmol} / \mathrm{paw}$, i.pl.), as well as DPCPX (10 and $30 \mathrm{nmol} / \mathrm{paw}$, i.pl.) with carbamazepine (600 nmol/paw) into the rat hind paw significantly decreased the antihyperalgesic effect of carbamazepine in a dose- and time-dependent manner (Fig. 2: B and C). The maximum inhibitory effects of 250 and $1000 \mathrm{nmol} /$ paw of caffeine on carbamazepine-induced antinociception were $53.4 \% \mathrm{I}$ and $79.4 \% \mathrm{I}$, respectively (not shown). The maximum inhibitory effects of 10 and $30 \mathrm{nmol} /$ paw of DPCPX on carbamazepine-induced antinociception were $58.3 \% \mathrm{I}$ and $85.2 \% \mathrm{I}$, respectively (not shown). The effects of carbamazepine, caffeine, and DPCPX were due to local effects, since they were not observed after administration of these drugs into the contralateral hind paw (Fig. 2: A, B, C). Peripheral coadministration of caffeine $(1000 \mathrm{nmol} / \mathrm{paw}, \mathrm{i} . \mathrm{pl}$.) or DPCPX (30 

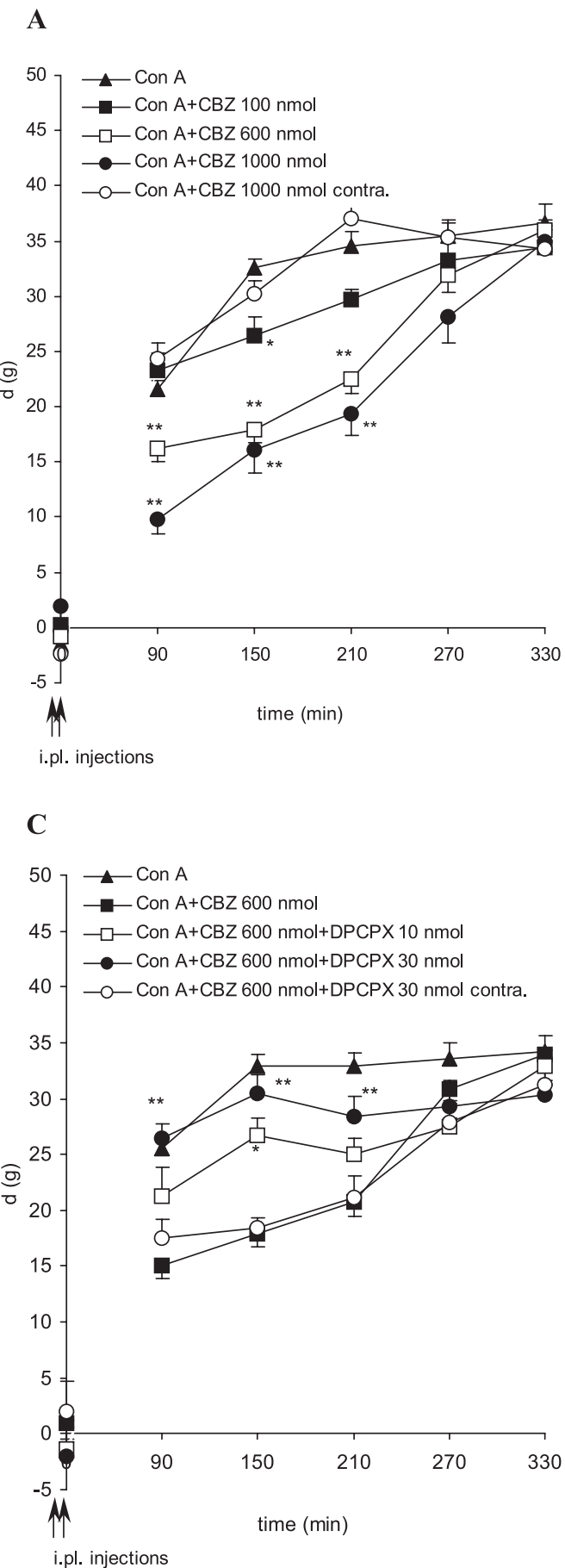

nmol/paw, i.pl.) with Con A failed to produce any significant effect on Con A-induced hyperalgesia ( $P>0.05$, Student's $t$-test, data not shown).

The present experiments revealed significant doseand time-dependent local peripheral antinociceptive action of carbamazepine in an inflammatory mechanical hyperalgesia model in the rat. The local nature of this action was verified by the lack of effect of carbamazepine injected into the contralateral hind paw. In

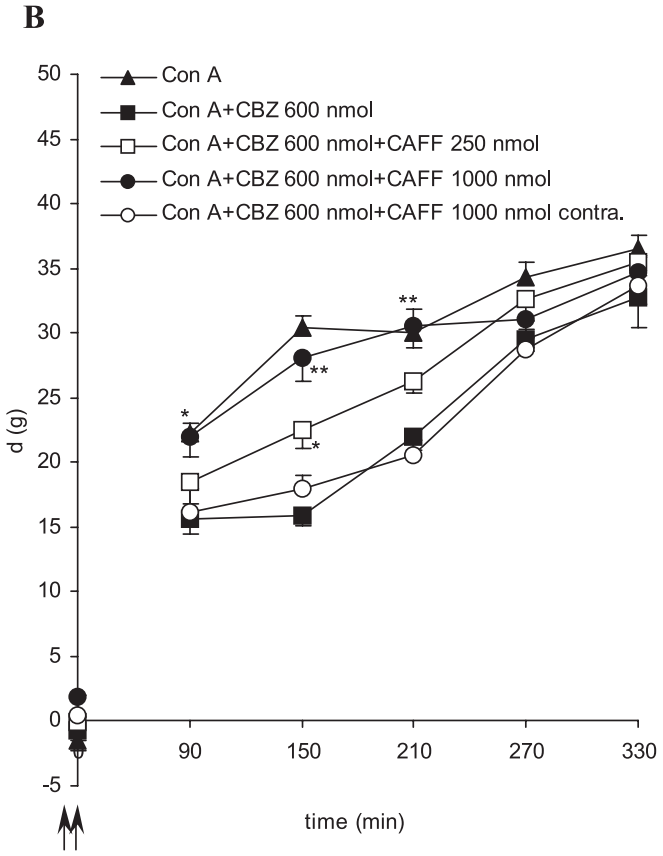

i.pl. injections

Fig. 2. Time course of the local peripheral antihyperalgesic effect of carbamazepine (CBZ) expressed as paw pressure differences in $g$ (d) between noninflamed and inflamed rat hind paw (A); inhibitory effect of local caffeine (CAFF) on local peripheral anti-hyperalgesic effect of CBZ expressed as d (g) (B); inhibitory effect of local DPCPX on local peripheral anti-hyperalgesic effect of CBZ expressed as d (g) (C). Predrug d (plotted at vertical axis) was obtained 10 min before intraplantar (i.pl.) injection (denoted by arrows). Each point represents the mean \pm S.E.M. of $d$ (g) obtained in 6-8 animals. Statistical significance $\left({ }^{*} P<0.05,{ }^{*} P<0.01\right.$; One-Way ANOVA followed by Tukey's HSD test) was determined by comparison with the curve for Con A alone (A) or Con A + CBZ $600 \mathrm{nmol}(\mathrm{B}, \mathrm{C})$. Contra. $=$ contralaterally.

our previous study, we demonstrated that systemically administrated carbamazepine produces antinociception in the same model, but whether the drug acts centrally and/or peripherally remained undetermined (4). In the present study, the significant local effects of carbamazepine $(100-1000 \mathrm{nmol} / \mathrm{paw}=0.12-1.18 \mathrm{mg}$ $/ \mathrm{kg}$, i.pl.) were attainable with much lower doses (up to 85 times lower) than the lowest effective systemic doses determined in our previous study $(10 \mathrm{mg} / \mathrm{kg}$, i.p. $)$. 
This could suggest that carbamazepine given systemically is able to achieve effective concentrations at the periphery and that there is considerable contribution of the peripheral antinociceptive effect to the net effect of systemically administered carbamazepine.

Earlier studies evaluated the antinociceptive potencies of carbamazepine administrated mostly by systemic and occasionally by a central route. Recently, some authors demonstrated that carbamazepine injected intraplantarly into the rat hind paw is a potent analgesic against acute thermal nociception in rats (11). The doses of carbamazepine they used were 3,000-6,000 times lower than the commonly used doses of lidocaine (1\% $2 \%$ solution or $1-2 \mathrm{mg} / 0.1 \mathrm{ml}$ ), a well known and widely used local anesthetic. However, our study provides the first evidence on the local peripheral antihyperalgesic effect of carbamazepine in the experimental model of inflammatory pain. The potency of carbamazepine in our study is about 700 times lower than that reported by another group (11) $\left(\mathrm{ED}_{50}\right.$ values: 0.21733 vs $0.00032 \mathrm{mg} / \mathrm{paw}$, respectively). By comparison, doses of carbamazepine that produce a systemic antinociceptive effect vary among studies that use different animal models of nociception from about 0.3 to $300 \mathrm{mg} / \mathrm{kg}(2-5,8)$.

It is well known that the purine nucleoside, adenosine, plays an important role in pain modulation. The adenosine $A_{1}$ receptor is the predominant receptor subtype mediating antinociception both peripherally and centrally, while the $A_{2 A}, A_{2 B}$, and $A_{3}$ receptors mediate nociception peripherally (6).

In the present experiments, the coadministration of nonselective adenosine $\mathrm{A}_{1}$ - and $\mathrm{A}_{2}$-receptor antagonist, caffeine (250 and $1000 \mathrm{nmol} / \mathrm{paw}$, i.pl.), with carbamazepine significantly depressed its local anti-hyperalgesic effect. As blockade of adenosine receptors is thought to underlie many of the pharmacological effects of caffeine (12), these observations suggest involvement of adenosine receptors in the anti-hyperalgesic effect of carbamazepine. However, the reversal of the antihyperalgesic effect of carbamazepine by a selective adenosine $\mathrm{A}_{1}$-receptor antagonist, DPCPX (10 and 30 nmol/paw, i.pl.), coadministrated with carbamazepine revealed that carbamazepine exerts its effect most probably through activation of adenosine $A_{1}$ receptors. This, taken together with previously reported data that carbamazepine binds to brain adenosine receptors $(7,13)$ and has no effect on adenosine uptake $(13,14)$, suggests direct interaction of carbamazepine with adenosine $A_{1}$ receptors, rather than an indirect action by enhancing adenosine tissue levels. Also, caffeine- and DPCPXinduced depressions are dose- and time-dependent and almost complete (up to $90 \%$ ) at the higher doses of antagonists used, speaking in favor of the direct adenosine receptor-mediated action of carbamazepine. Based on these findings, as well as on data that peripheral tissues possess adenosine $A_{1}$ receptors similar to those of central nervous system (15), and that activation of these receptors results in an antinociception in rats (6), we speculate that local peripheral anti-hyperalgesic effect of carbamazepine is mediated by direct activation of peripheral adenosine $A_{1}$ receptors by carbamazepine.

Earlier studies mainly suggested a blockade of sodium currents through voltage-gated sodium channels as a mechanism of the antinociceptive action of carbamazepine $(1,5,11)$. However, Mashimoto et al. demonstrated that the antinociceptive effect of carbamazepine in a tail flick test in stressed rats is mediated through activation of central adenosine $A_{1}$ receptors (8). Furthermore, the present results revealed that peripheral anti-hyperalgesic effect of carbamazepine is, at least partially, mediated by activation of peripheral adenosine $A_{1}$ receptors. This is in agreement with previous findings on the involvement of adenosine system in the mechanisms of antinociceptive action of carbamazepine $(4,8)$.

In conclusion, the results of the present study indicate that carbamazepine produces local peripheral antinociception against inflammatory pain, and peripheral $\mathrm{A}_{1}$ receptors might be a new target for the analgesic effect of carbamazepine.

\section{Acknowledgment}

This work was supported by the Ministry of Science and Environmental Protection of Serbia.

\section{References}

1 Ambrosio AF, Soares-da-Silva P, Carvalho CM, Carvalho AP. Mechanisms of action of carbamazepine and its derivatives, oxcarbazepine, BIA 2-093, and BIA 2-024. Neurochem Res. 2002;27:121-130.

2 Fox A, Gentry C, Patel S, Kesingland A, Bevan S. Comparative activity of the anti-convulsants oxcarbazepine, carbamazepine, lamotrigine and gabapentin in a model of neuropathic pain in the rat and guinea-pig. Pain. 2003;105:355-362.

3 Kiguchi S, Imamura T, Ichikawa K, Kojima M. Oxcarbazepine antinociception in animals with inflammatory pain or painful diabetic neuropathy. Clin Exp Pharmacol Physiol. 2004;31:5764.

4 Tomić MA, Vučković SM, Stepanović-Petrović RM, Ugrešić N, Prostran MS, Bošković B. The anti-hyperalgesic effects of carbamazepine and oxcarbazepine are attenuated by treatment with adenosine receptor antagonists. Pain. 2004;111:253-260.

5 Sakaue A, Honda M, Tanabe M, Ono H. Antinociceptive effects of sodium channel-blocking agents on acute pain in mice. $\mathrm{J}$ Pharmacol Sci. 2004;95:181-188.

6 Sawynok J, Liu XJ. Adenosine in the spinal cord and periphery: 
release and regulation of pain. Prog Neurobiol. 2003;69:313340.

7 Fujiwara Y, Sato M, Otsuki S. Interaction of carbamazepine and other drugs with adenosine $\left(\mathrm{A}_{1}\right.$ and $\left.\mathrm{A}_{2}\right)$ receptors. Psychopharmacology. 1986;90:332-335.

8 Mashimoto S, Ushijima I, Suetsugi M, Akimoto T, Watanabe K, Yamada M. Stress-dependent antinociceptive effects of carbamazepine: a study in stressed and nonstressed rats. Prog Neuropsychopharmacol Biol Psychiatry. 1998;22:159-168.

9 Vučković S, Prostran M, Ivanović M, Ristović Z, Stojanović R. Antinociceptive activity of the novel fentanyl analogue isocarfentanil in rats. Jpn J Pharmacol. 2000;84:188-195.

10 Tallarida RJ, Murray RB. Manual of pharmacologic calculations with computer programs. 2nd ed. New York, Berlin, Heidelberg, London, Paris, Tokyo: Springer Verlag; 1986.

11 Todorovic SM, Rastogi AJ, Jevtovic-Todorovic V. Potent analgesic effects of anticonvulsants on peripheral thermal nociception in rats. Br J Pharmacol. 2003;140:255-260.

12 Fredholm BB. Astra award lecture. Adenosine, adenosine receptors and the actions of caffeine. Pharmacol Toxicol. 1995;76:93101.

13 Skeritt JH, Davies LP, Johnston GA. A purinergic component in the anticonvulsant action of carbamazepine? Eur J Pharmacol. 1982;82:195-197.

14 Phillis JW. Interactions of the anticonvulsants diphenylhydantoin and carbamazepine with adenosine on cerebral cortical neurons. Epilepsia. 1984;25:765-772.

15 Alzheimer C, Kargl L, ten Bruggencate G. Adenosinergic inhibition in hippocampus is mediated by adenosine $\mathrm{A}_{1}$ receptors very similar to those of peripheral tissues. Eur $\mathrm{J}$ Pharmacol. 1991;196:313-317. 